\title{
The disc method
}

\section{John Erik Fornæss}

Mathematics Department, The University of Michigan, Ann Arbor, MI 48109, USA

(e-mail: fornaess@math.lsa.umich.edu)

Received 10 July 1996; in final form 26 August 1996

\section{Introduction}

The well known Bochner's Extension Theorem states that holomorphic functions defined in a connected neighborhood of the boundary of any bounded domain in $\mathbb{C}^{n}, n>1$, extend holomorphically to the interior of the domain.

There are three classical methods for proving such a result:

1: Using the Bochner-Martinelli kernel to extend functions to the inside.

2. To use solutions of $\bar{\partial}$ with compact support.

3. Pushing discs.

The first two methods are well established. The third method is folklore. The purpose of this paper is to show that contrary to folklore this method does not work. The above extension theorem cannot be proved by pushing discs step by step towards the interior. However, we are interested in proving the extension theorem in complex manifolds and, hence, would like to avoid pushing discs to the outside.

\section{Notation}

Let $\Omega$ be a bounded domain with connected $\mathcal{C}^{\infty}$ boundary in $\mathbb{C}^{2}$. Fix a small $\delta>0$, and consider the $\operatorname{ring} \tilde{\Omega}_{\delta}:=\{z \in \Omega ; \operatorname{dist}(z, \partial \Omega)<\delta\}$ where "dist" refers to Euclidean distance.

The $\epsilon$ Hartogs skeleton, $H_{\epsilon}$, is the domain

$$
\begin{aligned}
H_{\epsilon} & :=\left\{\left(z_{1}, z_{2}\right) ;\left|z_{1}\right|<1,\left|z_{2}\right|<\epsilon\right\} \\
& \cup\left\{1-\epsilon<\left|z_{1}\right|<1,\left|z_{2}\right|<1\right\} .
\end{aligned}
$$




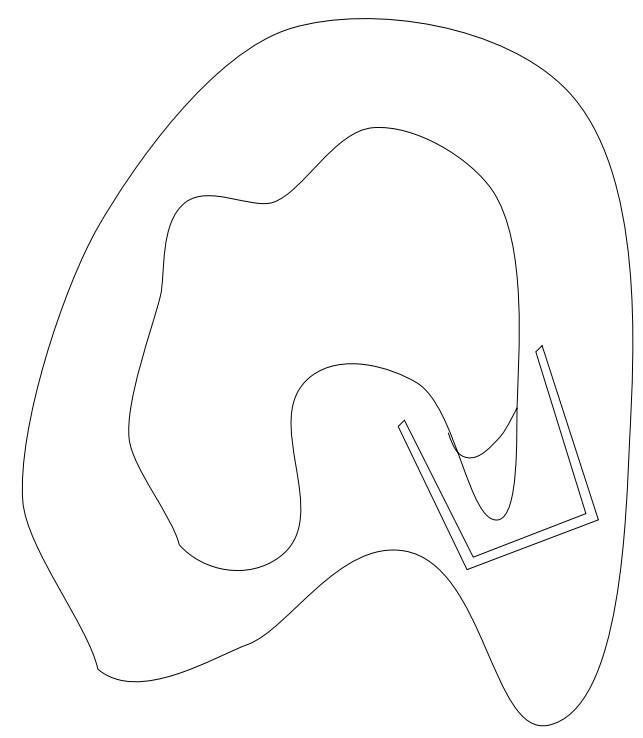

Fig. 1. Filling in of discs

The filled in Hartogs figure, $\hat{H}_{\epsilon}$, is the convex hull of $H_{\epsilon}$.

Definition 1 (See Figure 1.) We say that $\Omega$ can be filled in by pushing discs if there exists for any $\delta>0$ a finite sequence of connected domains with $\mathcal{C}^{\infty}$ boundary, $\tilde{\Omega}_{\delta}=\Omega_{1} \subset \Omega_{2} \subset \cdots \subset \Omega_{k}=\Omega$ and for each $j=1, \cdots, k-1$ there exists an $\epsilon_{j}>0$ and a univalent holomorphic map $\Phi_{j}$ defined on a neighborhood of $\hat{\hat{H}}_{\epsilon_{j}}$ such that

(1) $\Omega_{j+1} \subset \Omega_{j} \cup \Phi_{j}\left(\hat{H}_{\epsilon_{j}}\right) \subset \Omega$,

(2) $\Phi_{j}\left(H_{\epsilon_{j}}\right) \subset \Omega_{j}$

(3) $\Omega_{j} \cap \Phi_{j}\left(\hat{H}_{\epsilon_{j}}\right)$ is connected.

(4) $\Omega_{j+1} \cap \Phi_{j}\left(\hat{H}_{\epsilon_{j}}\right)$ is connected.

It is not too hard to prove that if $\Omega$ is pseudoconvex, $\Omega$ can be filled in by pushing discs. One can use a strictly plurisubharmonic exhaustion function which is also a Morse function and extend over sub-level sets via a bumping argument, taking particular care of the critical points of the exhaustion function.

\section{Construction of the example}

In this section we describe our example of a smoothly bounded domain $\Omega$ in $\mathbb{C}^{2}$ which cannot be filled in by pushing discs. 
Let $U_{1}:=\Delta^{2}$, the unit bidisc in $\mathbb{C}^{2}$. Consider four arcs in $U_{1}$ :

(1) $\gamma_{1}:=\left\{\left(x_{1}, 0,0,0\right) ;-1 \leq x_{1} \leq 0\right\}$

(2) $\gamma_{2}:=\left\{\left(0, i y_{1}, 0,0\right) ; 0 \leq y_{1} \leq 1 / 2\right\}$

(3) $\gamma_{3}:=\left\{\left(x_{1}, i / 2,0,0\right) ;-3 / 4 \leq x_{1} \leq 0\right\}$

(4) $\gamma_{4}:=\left\{\left(-3 / 4, i y_{1}, 0,0\right) ; 1 / 8 \leq y_{1} \leq 1 / 2\right\}$

Let $\gamma$ be a smooth curve obtained from $\gamma_{1} \cup \gamma_{2} \cup \gamma_{3} \cup \gamma_{4}$ by smoothing in a small neighborhood of each of the three corners.

Furthermore, we will attach a perturbation of a ball, with a hole drilled through, to the tip of $\gamma_{4}$. More precisely, let first $H^{\prime}$ be a pseudoconvex Hartogs domain of the form $H:=\left\{\left(z_{1}, z_{2}\right) \in \mathbb{C}^{2} ;\left|z_{1}+3 / 4\right|<1 / 8,\left|z_{2}\right|<\right.$ $\left.R\left(z_{1}\right)\right\}$ where $R$ is a radially symmetric function, $R>1 / 100$ and moreover, $R\left(z_{1}\right)=\sqrt{1 / 64-\left|z_{1}+3 / 4\right|^{2}}$ when $\left|z_{1}+3 / 4\right|<1 / 100$. In other words, on the top of the Hartogs figure, it is shaped like a ball, while at the edge it is shaped like a bidisc. Finally we remove, for some number $0<\rho<1 / 1000$, the tube $\left\{\left(x_{1}, i y_{1}, x_{2}, i y_{2}\right) ; y_{1}^{2}+x_{2}^{2}+y_{2}^{2}<\rho^{2}\right\}$ from $H$ and round off the corners in a very small neighborhood of the edges, where $\left|x_{1}+3 / 4\right|>$ $1 / 8-1 / 1000$ to create a (non-complete) smooth Hartogs domains $H_{\rho}$. For later, if $S$ is a set, let $S^{\delta}$ denote the tubular neigborhood about $S$ of radius $\delta$.

We attach $\gamma$ to $H$ and find a small tubular neighborhood of $\gamma$ with radius $0<\eta<\rho$ which is rounded off in a small neighborhood of the point $(-3 / 4, i / 8,0,0)$ to make a strongly pseudoconvex domain. Finally this neighborhood is rounded off in a small neighborhood of where it exits the bidisc $U_{1}$, which is also rounded off very near the distuinghuished boundary, in order to finish the construction of $\Omega=\Omega_{\rho, \eta}$ (See Figure 2). In other words, the inside of this tube and $H_{\rho}$ belongs to the exterior of $\Omega$. The domain $\Omega$ is obtained by drilling a hole into the rounded off bidisc. We can make $\rho$ and $\eta$ arbitrary if they are small enough.

For our construction we will also need the existence of a smooth function $\sigma_{\rho, \eta}=\eta \leq \sigma\left(x_{1}\right) \leq \rho$ defined for $-7 / 8+1 / 1000<x_{1}<-5 / 8-$ $1 / 1000$ such that $\sigma\left(x_{1}\right) \equiv \rho, x_{1} \in(-7 / 8+1 / 1000,-7 / 8+2 / 1000)$ while $\sigma\left(x_{1}\right) \equiv \eta, x_{1} \in(-5 / 8-2 / 1000,-5 / 8-1 / 1000)$. Moreover, we need that the tube $T=\left\{\left(x_{1}, i y_{1}, x_{2}, i y_{2}\right) ; x_{1} \in(-7 / 8+1 / 1000,-5 / 8-\right.$ $1 / 1000), y_{1}^{2}+x_{2}^{2}+y_{2}^{2}<\sigma^{2}\left(x_{1}\right)$ is strongly pseudoconvex. This is surely possible as long as $\eta$ and $\rho$ are close enough together. Observe furthermore, that if $\eta$ and $\rho$ are close enough and $\delta>0$ is any sufficiently small number, $\eta+\delta<\rho-\delta$, then $\sigma_{\rho-\delta, \eta+\delta}$ exists also. 


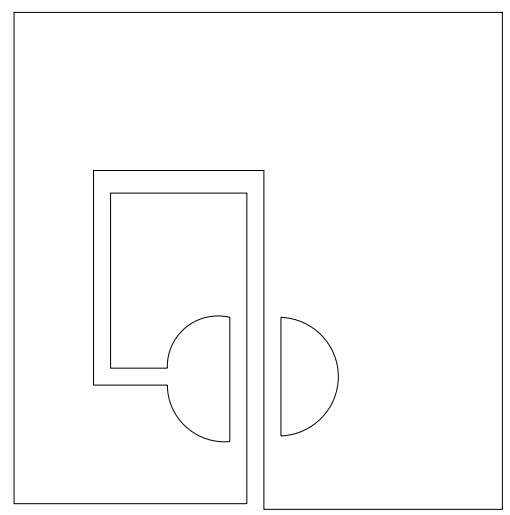

Fig. 2. The example which cannot be filled in by discs

\section{Proof that the example works}

We construct first a barrier domain for pushing discs. This barrier domain is a double sheeted Riemann domain, $\mathcal{R}_{\delta}$, over $\mathbb{C}^{2}$. To describe this Riemann domain, we will describe three open sets and explain how points in them are identified to make a Riemann domain. Pick any small $\delta>0$. One piece, $U_{1}$, of our Riemann domain will consist of the open sheet between $\partial \Omega$ and $\partial \tilde{\Omega}_{\delta}$ and which lies next to $H_{\rho}$ as well as $\gamma$ except for the part of $\gamma_{1}$ where $x_{1}<-5 / 8-1 / 1000$. Over the piece of $\gamma_{1}$, where $-7 / 8+1.5 / 1000<$ $x_{1}<-5 / 8-1 / 1000$, the sheet is given by those points in $\Omega$ for which $y_{1}^{2}+x_{2}^{2}+y_{2}^{2}<\sigma_{\rho-\delta, \eta+\delta}^{2}$.

The next piece of the Riemann domain, $U_{2}$, consists of the points in $\Omega$ of distance at most $\delta$ from $H$. Then $U_{1} \cap U_{2}$ contains two connected components $V_{1}, V_{2}$. The set $V_{1}$ is the component containing the narrow hollow tube about the $x_{1}-$ axis. We identify $U_{1}$ and $U_{2}$ over $V_{2}$ only. So $U_{1}$ and $U_{2}$ together defines a two-sheeted Riemann domain.

The third piece, $U_{3}$, consists of all points in $\Omega$ after taking out $\overline{U_{1} \cup U_{2}}$ and adding in again those points in $H^{\delta} \backslash \overline{H_{\rho}^{\delta}}$ for which $x_{1}<-7 / 8+2 / 1000$. Then the points in $U_{1}$ and $U_{3}$ are identified along $U_{1} \cap U_{3}$. In fact $U_{1} \cup U_{3}$ is a schlicht piece of $\mathcal{R}_{\delta}$. Observe that $\tilde{\Omega}_{\delta} \subset U_{1} \cup U_{3}$.

Suppose that $\Omega$ can be filled in by pushing discs. Let $\Omega_{1}, \cdots, \Omega_{k}$ be as in Definition 2.1. We will show inductively that each $\Omega_{j}$ can be lifted to $\mathcal{R}_{\delta}$. This will finish the proof because many points of $\Omega$ are not in the projection of $\mathcal{R}_{\delta}$.

At first we observe that $\Omega_{1}=\tilde{\Omega}_{\delta}$ and hence can be lifted to $\mathcal{R}_{\delta}$. Suppose next that $j<k$ and that $\Omega_{j}$ can be lifted to $\mathcal{R}_{\delta}$. Suppose that $\Omega_{j+1}$ cannot be lifted to $\mathcal{R}_{\delta}$. Consider the Hartogs figure $\Phi_{j}\left(\hat{H}_{\epsilon_{j}}\right)$. There must then be a smallest $\left|z_{2}\right|,\left|z_{2}^{0}\right|$, so that the image $D$ of the disc $\left.\left\{z_{1}, z_{2}^{0}\right) ;\left|z_{1}\right|<1\right\}$ 
intersects the boundary of $\mathcal{R}_{\delta}$. The boundary of this disc $D$ is in $\mathcal{R}_{\delta}$. However the interior of the disc hits the boundary of $\mathcal{R}_{\delta}$. We will show that this is impossible by considering the different boundary points of $\mathcal{R}_{\delta}$. First, consider the boundary points of $\mathcal{R}_{\delta}$ which are also boundary points of $U_{1}$. Those points which are on the boundary of $\Omega$ are excluded. The remaining points are all strongly pseudoconvex however, so they are ruled out. Next consider the boundary points of $\mathcal{R}_{\delta}$ which are boundary points of $U_{2}$. But these are either points of $\partial \Omega$, which are excluded, or they belong to the flat part of the boundary of $H^{\delta}$ over the boundary of the disc $\left|z_{1}+3 / 4\right|=1 / 8+\delta$. Again we get a contradiction from pseudoconvexity.

Finally consider the boundary points of $\mathcal{R}_{\delta}$ which are boundary points of $U_{3}$. The boundary points of $\Omega$ are again excluded. The other boundary points are either boundary points of $U_{1}$, which are excluded because all points on the other side are already in $\Omega_{j}$, contradicting condition (3) of Definition 2.1 , or the points are in the flat part of the boundary of $H^{\delta}$ over the disc $\left|z_{1}+3 / 4\right|=1 / 8$, but these cannot be reached either by pseudoconvexity considerations. 